\title{
ROLE OF ANTIMICROBIALS IN INTRA OCULAR LENS (IOL) IMPLANTATION
}

Patil Banderao V1, Ashok Binjawadgi ${ }^{2}$, Prashant Dass ${ }^{3}$.

1. Professor, Department of Pharmacology, MR Medical College, Gulbarga.

2. Associate Professor, Department of Pharmacology, MR Medical College, Gulbarga.

3. Post Graduate Resident, Department of Pharmacology, MR Medical College, Gulbarga.

\section{CORRESPONDING AUTHOR}

Dr. Patil Banderao V,

Professor, Department of Pharmacology,

MR Medical College,

Gulbarga, Karnataka- 585105.

Email-drpatilbv@gmail.com

\section{HOW TO CITE THIS ARTICLE:}

Patil Banderao V, Ashok Binjawadgi, Prashant Dass. "Role of antimicrobials in intra ocular lens (iol) implantation". Journal of Evolution of Medical and Dental Sciences 2013; Vol2, Issue 26, July 1; Page: 48524860.

ABSTRACT: - CONTEXT: Pharmacotherapy which means use of drugs for prevention and treatment of diseases is a major branch of therapeutics. Cataract, particularly in the age group of 40-75 years is fairly common.

Many potent drugs like Glucocorticoids, Antimicrobials etc. are used in the management of cataract. Antimicrobials use has led to a big concern amongst clinicians as many consider it to be unnecessary, inappropriate and downright dangerous.

Recently increased attention has been diverted to rational prescribing and drug utilisation studies play a major role in this regard. These studies not only detect flaws in the therapy, but also help to find out solution to rectify the same.

Anti-microbials are prescribed for almost all cases posted for Intra Ocular Lens Implantation Surgery. Hence the present prospective observational study was undertaken with respect to the use of anti-microbials (both prophylactic and post-operative) in Ophthalmology practice.

\section{AIMS}

1. A 'Hospital Based Study' with an aim to carry out a complete therapeutic audit and see what is prescribed and what is the intention.

2. To study the role of anti-microbials during IOL implantation.

3. To study the prophylactic role of anti-microbials during IOL implantation.

4. To suggest measures to rationalise the therapeutic regimen.

METHODS AND MATERIALS: Two teaching hospitals attached to MR Medical College, Gulbarga were selected, Government General Hospital and HKES Basaveshwar Teaching Hospital.

The study was carried out from 01-08-2012 to 30-04-2013 after due permission from the Institutional Ethics Committee, Head of Department of Ophthalmology, Basaveshwar Teaching Hospital and the Dean, MR Medical College.. 


\section{ORIGINAL ARTICLE}

RESULTS AND CONCLUSION: Our study found that Moxifloxacin is the most frequently prescribed anti-microbial pre-operatively for IOL implantation. Also, Moxifloxacin is the most frequently prescribed anti-microbial post-operatively for IOL implantation.

Overall Moxifloxacin is currently the most frequently prescribed anti-microbial for both pre and post-operative use in IOL implantation surgery.

KEY WORDS: Anti-microbials, Drug Utilization Study, Intra ocular lens, Cataract, Prophylactic, Postoperative

INTRODUCTION: The subject of therapeutics is the first of the medical sciences to come into existence. From birth to death, man is exposed to a variety of diseases.. Pharmacotherapy which means use of drugs for prevention and treatment of diseases is a major branch of therapeutics. ${ }^{1,2}$ Cataract, particularly in the age group of 40-75 years is fairly common ${ }^{3}$.

Many potent drugs such as Glucocorticoids ${ }^{4}$, Antimicrobials ${ }^{5}$ etc. are used in the management of cataract. Antimicrobials use has led to a big concern amongst clinicians, especially whence many consider it to be unnecessary, inappropriate and downright dangerous ${ }^{6}$. Hence there is a need for a systematic review regarding the role of antimicrobials in such a setting.

Prescription is a legal written order containing drugs given by the physicians to the patients. Errors in prescription may be due to inadequate knowledge about the disease ${ }^{7}$ or the drug prescribed $^{8}$ or ignorance ${ }^{9}$. So it should be scientifically legible, unambiguous, adequate and complete. It has been well accepted that inadequate and irrational prescriptions could lead to serious consequences ${ }^{10}$.

The prescriber must remember that a scientific approach does not mean a patient as a mere biological machine but treating the spiritual, psychological and social dimensions of human beings in a rational manner.

Errors in prescription are not uncommon and could be due to ignorance ${ }^{5}$ or inadequate knowledge about the disease ${ }^{6}$ and pharmacology of the drugs prescribed. ${ }^{7}$ Erroneous prescriptions are recognised even in the tertiary care hospital. ${ }^{8}$

Recently an increased attention has been diverted to rational prescribing and drug utilisation studies play a major role in this regard. ${ }^{9}$ These studies not only detect flaws in the therapy, but also help to find out solution to rectify the same. ${ }^{10}$

The present study was undertaken to analyse and systematically review the role of antimicrobials given to patients undergoing intra ocular lens (IOL) implantation surgery in Government General Hospital and Basaveshwar Teaching Hospital, Gulbarga, Karnataka.

OBJECTIVES: The present study was conducted in Government General Hospital and Basaveshwar Teaching Hospital keeping the following aims and objectives in mind:

1. A 'Hospital Based Study' with an aim to carry out a complete therapeutic audit and see what is prescribed and what is the intention.

2. To study the role of antimicrobials during IOL implantation.

3. To study the prophylactic role of antimicrobials during IOL implantation.

4. To suggest measures to rationalise the therapeutic regimen. 


\section{ORIGINAL ARTICLE}

MATERIALS AND METHODS: The present study was undertaken to analyse and systematically review the role of antimicrobials given to patients undergoing intra ocular lens (IOL) implantation surgery .Two teaching hospitals attached to MR Medical College, Gulbarga were selected, namely Government General Hospital and HKES Basaveshwar Teaching Hospital. They represented both upper and lower economic strata of the population.

The study was carried out from 01-08-2012 to 30-04-2013 after due permission from the Institutional Ethics Committee, Head of Department of Ophthalmology, Basaveshwar Teaching Hospital and the Dean, MR Medical College. A Proforma and an Informed Consent form were provided to all the participating doctors.

All the prescriptions were analysed and classified according to the antimicrobial agent employed.

The present study included all patients who were admitted for IOL implantation surgery between the ages of 40-75 years of either sex. Patients were selected randomly and detailed history was elicited from all. Patients with multiple, concomitant illnesses such as diabetes mellitus, hypertension, tuberculosis etc. were excluded from the study.

A total of 104 prescriptions were collected, analysed and classified. The prescriptions were collected daily, right from the day of admission till the time of discharge of the patient. Antimicrobial agents prescribed during the hospital stay and at the time of discharge were also noted.

The data collection of patients subjected to IOL implantation was done between 10AM-12PM and 4PM-6PM. The morning time was so chosen because most of the patients were usually discharged post morning rounds and checkup.

DATA ANALYSIS: As mentioned above, 104 prescriptions were collected and analysed. This data was further condensed and a master chart was prepared using MS-Excel. The data was subjected to statistical analysis.

The overall information generated was presented under the following headings:

1. Age wise distribution of cataract in patients undergoing IOL implantation surgery.

2. Sex wise distribution of cataract in patients undergoing IOL implantation surgery.

3. Distribution of patients according to the type of cataract involved.

4. List of all antimicrobial agents employed in patients undergoing IOL implantation surgery.

5. Pre-operative antimicrobial agents employed in patients undergoing IOL implantation surgery.

6. Post-operative antimicrobial agents employed in patients undergoing IOL implantation surgery.

7. Comparison of prescribed dose with established standard dose.

RESULTS: About 104 prescriptions were included in the study and analysed after matching the inclusion and exclusion criteria as mentioned earlier from 01-08-2012 to 30-04-2013 from Government General Hospital and Basaveshwar Teaching and General Hospital.

Out of the 104 prescriptions studied:

1. Maximum numbers of patients were encountered in the age group of 51-60 years.

2. $48.07 \%$ were male and $51.92 \%$ were females

3. A whopping $91.35 \%$ of cases for IOL implantation were having mature senile cataract. 


\section{ORIGINAL ARTICLE}

4. Antimicrobial agents were used topically.

5. Pre-operative antimicrobials employed included drugs such as Moxifloxacin, Gentamicin and Ciprofloxacin.

6. Post-operative antimicrobials employed included drugs such as Moxifloxacin, Gentamicin, Ciprofloxacin, Tobramycin, Ofloxacin and Gatifloxacin.

7. Our comparison stated that Moxifloxacin is the most frequently prescribed anti-microbial pre-operatively for IOL implantation.

8. Also, Moxifloxacin is the most frequently prescribed anti-microbial post-operatively for IOL implantation.

9. Overall Moxifloxacin is currently the most frequently prescribed anti-microbial for both pre and post-operative use in IOL implantation surgery.

TABLE-1- Age wise distribution of cataract in patients undergoing IOL implantation surgery Total number of cases $=104$

\begin{tabular}{|l|l|l|}
\hline Age in Years & Number of Patients & Percentage according to age \\
\hline $41-50$ & 25 & 24.03 \\
\hline $51-60$ & 36 & 34.61 \\
\hline $61-70$ & 31 & 29.80 \\
\hline $71-80$ & 12 & 11.53 \\
\hline
\end{tabular}

TABLE-2- Sex wise distribution of cataract in patients undergoing IOL implantation surgery Total number of cases $=104$

\begin{tabular}{|l|l|l|}
\hline Sex & Number of Patients & Percentage according to sex \\
\hline Male & 50 & 48.08 \\
\hline Female & 54 & 51.92 \\
\hline
\end{tabular}

TABLE-3- Distribution of patients according to the type of cataract involved Total number of cases = 104

\begin{tabular}{|l|l|l|}
\hline Indication & Number of Patients & $\begin{array}{l}\text { Percentage of types of } \\
\text { cataract }\end{array}$ \\
\hline Senile mature cataract & 95 & 91.35 \\
\hline Developmental cataract & 4 & 3.85 \\
\hline Complicated cataract & 2 & 1.92 \\
\hline Traumatic cataract & 2 & 1.92 \\
\hline Aphakia & 1 & 0.96 \\
\hline
\end{tabular}

List of all antimicrobial agents employed in patients undergoing IOL implantation surgery

1. Moxifloxacin

2. Gentamicin

3. Ciprofloxacin

4. Tobramycin

5. Ofloxacin

6. Gatifloxacin 
TABLE-4- List of antimicrobial agents employed pre-operatively in patients undergoing IOL implantation surgery Total Number of Cases $=104$

\begin{tabular}{|l|l|l|}
\hline Drug & Number of Patients & $\begin{array}{l}\text { Percentage of Anti-microbials } \\
\text { for pre-operative use }\end{array}$ \\
\hline Moxifloxacin $0.5 \%$ & 70 & $67.3 \%$ \\
\hline Gentamicin $0.3 \%$ & 28 & $26.92 \%$ \\
\hline Ciprofloxacin $0.3 \%$ & 6 & $5.78 \%$ \\
\hline
\end{tabular}

Table-5- List of antimicrobial agents employed post-operatively in patients undergoing IOL implantation surgery

Total Number of Cases $=104$

\begin{tabular}{|l|l|l|}
\hline Drug & Number of Patients & $\begin{array}{l}\text { Percentage of Anti-microbials } \\
\text { for post-operative use }\end{array}$ \\
\hline Moxifloxacin $0.5 \%$ & 72 & $69.23 \%$ \\
\hline Gentamicin $0.3 \%$ & 4 & $3.8 \%$ \\
\hline Ciprofloxacin $0.3 \%$ & 8 & $7.69 \%$ \\
\hline Tobramycin $0.3 \%$ & 12 & $11.53 \%$ \\
\hline Ofloxacin $0.3 \%$ & 6 & $5.76 \%$ \\
\hline Gatifloxacin $0.3 \%$ & 2 & $1.92 \%$ \\
\hline
\end{tabular}

DISCUSSION: Cataract is a leading cause of blindness worldwide, and India alone has an estimated 8 million people who are blind due to cataract with an annual increase upto 3 million. Surgical Intraocular Lens implantation is the best treatment option currently available.

The aim of the study was to throw light on the aspects like surgeons' preference for a particular anti-microbial, the drug utilization pattern, a comparison between the standard dose and the prescribed dose, to find out irrationality amongst the prescriptions and to suggest methods for rationalisation of existing therapeutic modalities. The selection of a particular anti-microbial in the clinical practice of Ophthalmology is difficult because of the advent of newer and safer drugs like Moxifloxacin, Tobramycin and Gatifloxacin over the recent past 11.

The study was done over a period of 8 months, from 01-08-2012 to 30-04-2013 involving a total of 104 patients posted for IOL implantation. All patients in our study were discharged the same day of surgery and no hospital stay was required.

The results of our study indicated that the maximum number of patients was encountered in the age group of 51-60 years amounting to approximately $34 \%$ of the total study population (Table 1). Cataract is a fairly common ailment in the elderly and $75 \%$ of our study population was above 50 years of age.

Sex of the patient did not play a vital role and almost all the cases were equally divided amongst both the sexes (Table 2).

Maximum indication for IOL implantation surgery in the present study was for senile mature cataract. A whopping $91.35 \%$ of cases for IOL implantation were having mature senile cataract, whereas $3.85 \%$ patients had developmental cataract, $1.92 \%$ had traumatic cataract and $0.96 \%$ suffered from aphakia (Table 3). This reiterates that senile mature cataract is currently the most common indication for IOL implantation surgery. 


\section{ORIGINAL ARTICLE}

Our present study found out that all 104 patients studied were prescribed topical antimicrobials. These were employed both pre and post-operatively. Pre-operative (prophylactic) antimicrobials employed included drugs such as Moxifloxacin, Gentamicin and Ciprofloxacin. Amongst them, Moxifloxacin 0.5\%, was employed in $67.3 \%$ of the cases making it the overwhelmingly favourite choice of the clinicians currently (Table 4). Older drugs like Gentamicin $0.3 \%$ has been losing favour amongst clinicians owing to its side effect profile and was prescribed only in $26.92 \%$ of the cases.

Post-operative antimicrobials employed included drugs such as Moxifloxacin, Gentamicin, Ciprofloxacin, Tobramycin, Ofloxacin and Gatifloxacin. Here too, Moxifloxacin $0.5 \%$ was the overwhelmingly favoured drug, garnering a share of $69.23 \%$. Tobramycin $0.3 \%$ was the next most favoured anti-microbial agent and was prescribed in approximately $11 \%$ of the study population (Table 5).

In the present study, a few prescriptions were found to be irrational. The WHO draft of 1983 describes the criteria for irrational drug prescription as, the medication prescribed is incorrect, unnecessary, inadequate, inappropriate or excessive ${ }^{12}$. Anti-microbial prescriptions were rational in case of pre-operative use of these drugs. The problem arose in a few cases in the daily dosing schedule of Tobramycin $0.3 \%$, Ofloxacin $0.3 \%$ and Gatifloxacin $0.3 \%$ when used in the postoperative setting. Two cases out of twelve who were administered Tobramycin and one out of six administered Ofloxacin were using the drug twice a day instead of the standard regimen of six hourly administration ${ }^{13}$. One person was incorrectly administered Gatifloxacin three times a day against the standard regimen of six hourly dosing ${ }^{14}$.

CONCLUSION; Our study found that Moxifloxacin is the most frequently prescribed anti-microbial pre-operatively for IOL implantation. Also, Moxifloxacin is the most frequently prescribed antimicrobial post-operatively for IOL implantation.

Overall Moxifloxacin is currently the most frequently prescribed anti-microbial for both pre and post-operative use in IOL implantation surgery.

Irrational prescriptions are harmful because they lead to a number of problems such as increased cost of the therapy, therapeutic failure, adverse drug reactions, dangerous drug-drug interactions etc. One way of promoting rational prescribing is by conducting drug utilisation studies, educating and training the doctors adequately regarding the need for rational prescribing.

\section{REFERENCES:}

1. DiPiro, Joseph T, Talbert RL et al. Pharmacotherapy: a pathophysiologic approach. New York, NY: McGraw-Hill, 2005.

2. Peuskens J. The evolving definition of treatment resistance. Journal of Clinical Psychiatry, 1999.

3. KleinBE, Klein R, Lee KE. Incidence of age-related cataract: the Beaver Dam Eye Study. Archives of Ophthalmology; 1998: 116(2), 219.

4. Shah GK., Stein JD, Sharma S et al. Visual outcomes following the use of intravitreal steroids in the treatment of postoperative endophthalmitis. Ophthalmology 107, no. 3; 2000: 486489. 
5. Seppala H, Mohammad AJ, Järvinen H et al. Effect of prophylactic antibiotics on antimicrobial resistance of viridans streptococci in the normal flora of cataract surgery patients. Journal of Cataract \& Refractive Surgery 30, no. 2; 2004: 307-315.

6. Shiva F, Eidikhani A L, Padyab M. Prescription practices in acute pediatric infections. Journal of Pediatric Infectious Diseases; 2006: 1 (1), 25-28.

7. Aronson JK. Medication errors: what they are, how they happen, and how to avoid them. QJM; 2009: 102(8), 513-521.

8. McDowell SE, Ferner HS, Ferner RE. The pathophysiology of medication errors: how and where they arise. British Journal of Clinical Pharmacology; 2009: 67, no. 6: 605-613.

9. Tully MP, Ashcroft DM, Dornan T et al. The causes of and factors associated with prescribing errors in hospital inpatients. Drug Safety; 2009: 32(10), 819-836.

10. Mahar, Michael L. Prescription order packaging system and method. U.S. Patent 6,769,228, issued August 3, 2004.

11. Liesegang TJ. Use of antimicrobials to prevent postoperative infection in patients with cataracts. Current opinion in Ophthalmology 12, no. 1; 2001: 68-74.

12. De Vries CS, ThFJ de T,Blijleven W. Prescription data as a tool in pharmacotherapy audit (I) general considerations. Pharmacy World and Science 21, no. 2; 1999: 80-84.

13. CochereauI, Amel M, Moncef $\mathrm{K}$ et al. 3-day treatment with azithromycin $1.5 \%$ eye drops versus 7 -day treatment with tobramycin $0.3 \%$ for purulent bacterial conjunctivitis: multicentre randomised and controlled trial in adults and children. British Journal of Ophthalmology 91, no. 4; 2007: 465-469.

14. Yee RW, Tepedino $\mathrm{M}$, Bernstein $\mathrm{P}$ et al. A randomized, investigator-masked clinical trial comparing the efficacy and safety of gatifloxacin $0.3 \%$ administered BID versus QID for the treatment of acute bacterial conjunctivitis. Current Medical Research and Opinion ${ }^{\circledR} 21$, no. 3; 2005: 425-431.

\section{CHART-1}

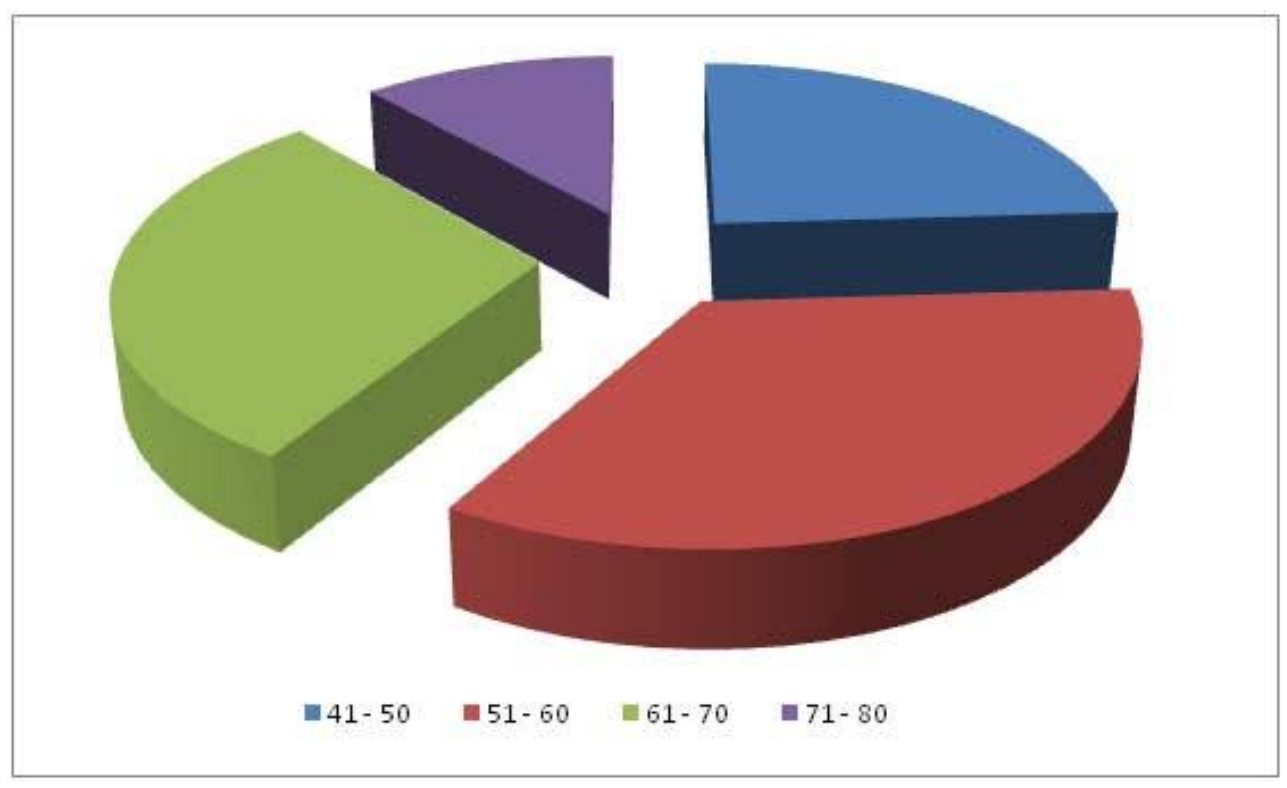




\section{ORIGINAL ARTICLE}

\section{CHART-2}

\section{Sex wise Distribution (in \%)}

- Male Female
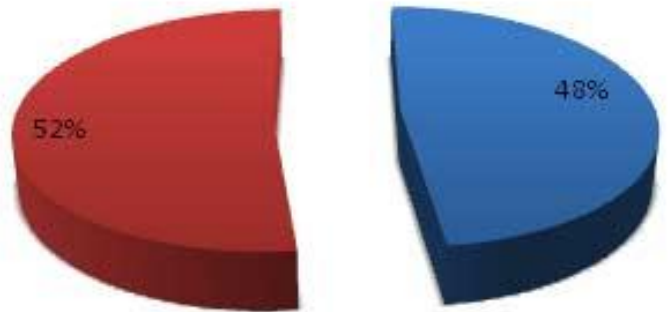

\section{CHART-3}

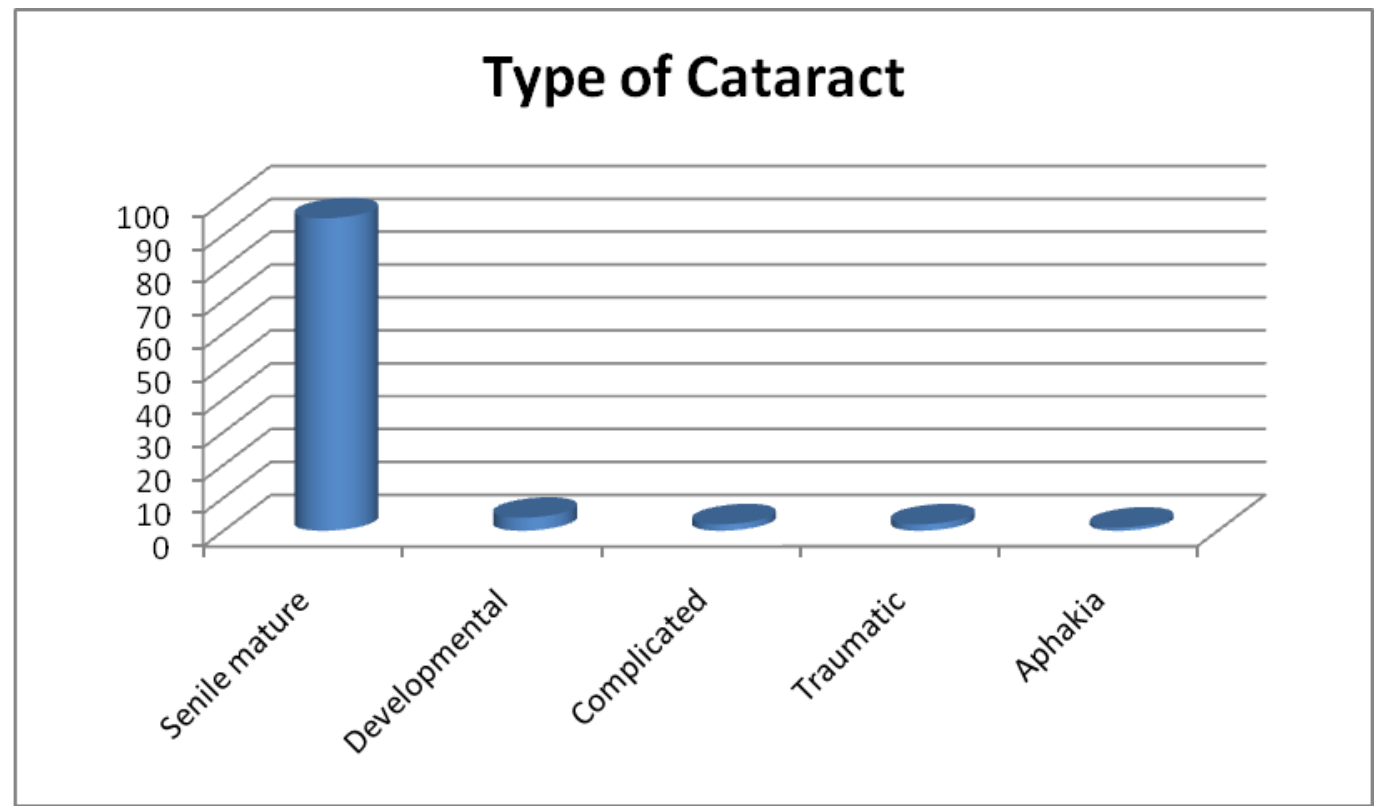




\section{ORIGINAL ARTICLE}

\section{Chart-4}

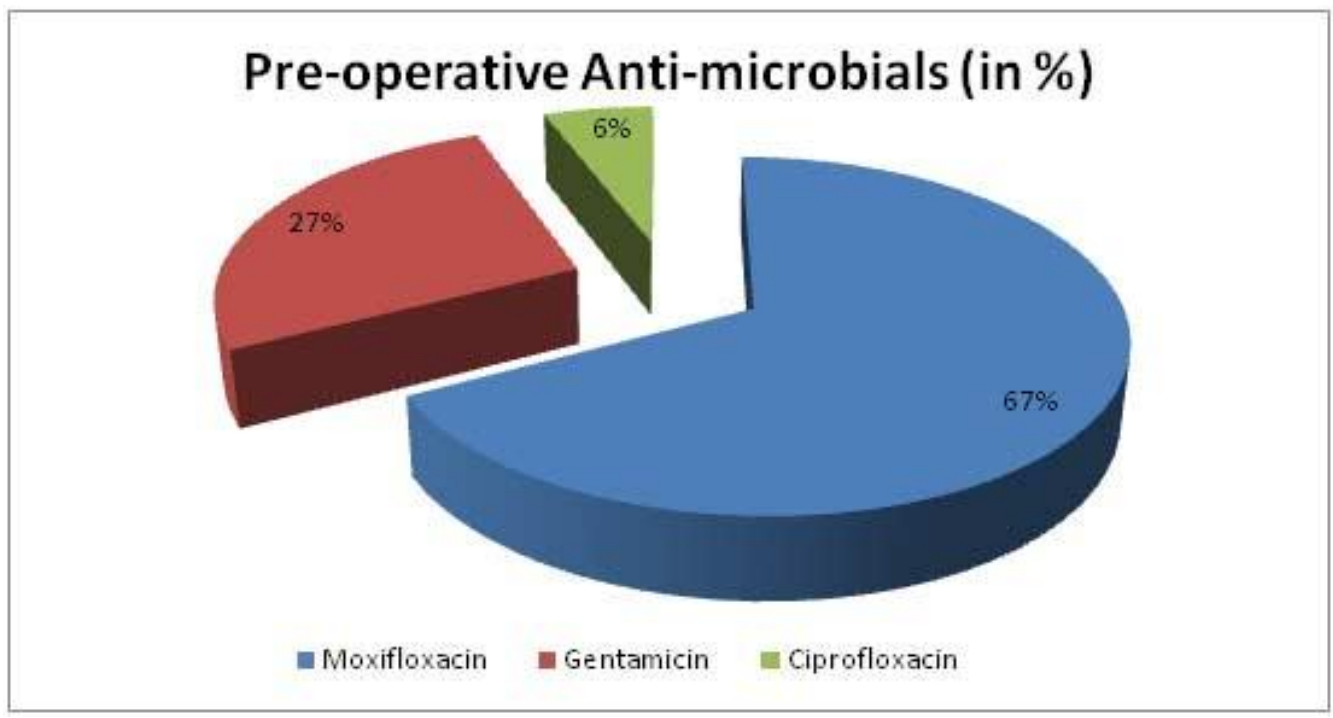

\section{Chart-5}

\section{Post-operative Anti-microbials (in \%)}

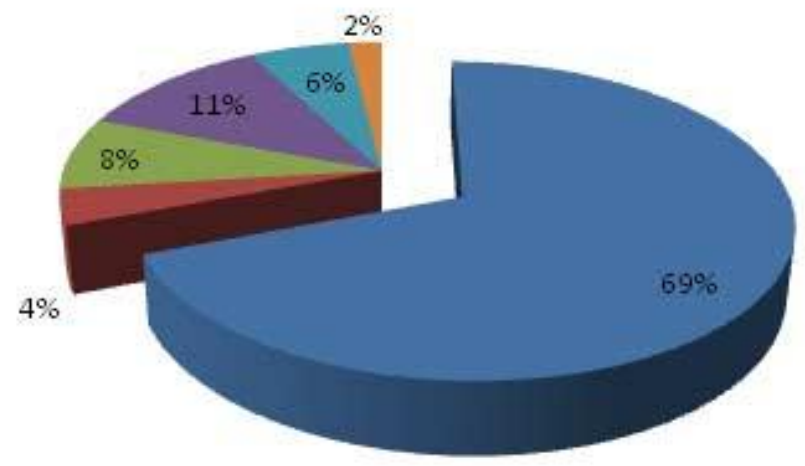

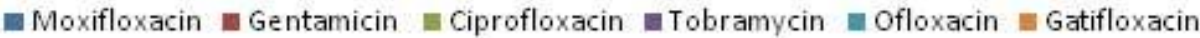

